\title{
Promotion of Copper Corrosion Inhibition by Application of a Square Wave Potential Regime to Copper Specimens in Polyvinylpyrrolidone Solutions
}

\author{
Mohammed K. Hourani ${ }^{a,{ }^{*}}$ and Hamzah Abo-Hassan ${ }^{b}$ \\ ${ }^{a}$ Department of Chemistry, Electrochemistry Research Laboratory, University of Jordan, \\ Amman 11942, Jordan \\ ${ }^{b}$ Royal Scientific Society, Amman 11942, Jordan
}

Received April 20, 2016; accepted July 15, 2016

\begin{abstract}
Enhancement of corrosion inhibition of copper by polyvinylpyrrolidone (PVP) upon application of a square wave potential regime to copper specimens was investigated. The results indicated a marked decrease of copper corrosion rate upon application of a square wave to copper specimens in PVP - containing solutions. The inhibition enhancement was found to increase when increasing PVP concentration, and with a larger time of exposure with application of the square wave potential regime. A $500 \mathrm{~Hz}$ frequency was the optimal frequency for promotion of inhibition by PVP. SEM micrographs revealed that application of the square wave converted the dendritic coverage of PVP to a more uniform layer of PVP at copper surface. The enhancement of corrosion inhibition by application of the square wave might be explained on basis of the enhanced adsorption of PVP onto copper surface by application of the square wave potential regime. Application of square wave potential regime apparently influences the PVP adsorption coverage at the copper surface.
\end{abstract}

Keywords: corrosion of copper, corrosion inhibition, square wave potential regimes, promotion of corrosion inhibition.

\section{Introduction}

Square wave, in a sense, is a train of double-potential step experiments [1]. The double-potential step comprises a forward step and a reverse step, which means that it involves a process, and though not necessarily its reversal, in each complete cycle [2]. Applications of the square wave to induce changes at the electrode surface have been reported for more than two decades [2,3,4]. Preparation of preferentially oriented metallic surfaces by application of a square wave of potential regimes has been reported $[2,3,4]$, and their use for preparation of electrocatalysts has been reported too [2]. In our laboratory, we have proved the notion that square wave potential regimes can be used for elimination of

\footnotetext{
*Corresponding author. E-mail address: mhourani@ju.edu.jo
} 
some pollutants [5], and desulfurization of thiophene as a model for sulfur compounds [6]. Later on, we have shown that square wave potential regimes with suitable parameters can be used for preparation of nano and microstructured metallic surfaces $[7,8]$.

Corrosion, on the other hand, is an electrochemical process [9], and corrosion inhibition, to a great extent, depends on the surface coverage and the state on which the inhibitor exists at the surface of the metallic or alloy substrate [10]. Orientation or polymerization of the inhibitor at the metal surface may affect the rate of corrosion, i.e., promote corrosion resistance of the metal.

Polyvinylpyrrolidone (PVP) is a nontoxic, environment friendly water soluble polymer [11]. These properties of PVP render this polymer an interesting material for green corrosion inhibition [12]. Moreover, polyvinylpyrrolidone exhibits the requirements that must be found in a typical inhibitor. PVP contains the oxazole moiety, an N-heterocycle, where N-heterocyclic compounds are well-known for their efficient inhibition of copper corrosion in a solution containing chloride [13]. Heteroatoms, $\pi$ bonds and aromatic rings are three factors which contribute to the efficiency of the adsorption of N-heterocyclic compounds onto copper surfaces, and, consequently, corrosion inhibition of copper [14]. PVP, in addition to containing the oxazole moiety, is a polymer which facilitates the formation of a protective adsorbed layer on the surface copper by the inherent cross-linking of the oxazole, $\mathrm{N}$-heteroatomic moieties [15]. Thus, the protective polyvinylpyrrolidone formed at the copper surface blocks the copper active sites and hinders the electrochemical communication between the solution and copper atoms [16]. For this reason, polyvinylpyrrolidone was chosen as a model for the inhibitor, while copper was chosen as the model for a corroding metal for the exploration of the effect of the application of square wave potential regimes on the rate of copper corrosion.

\section{Experimental section}

\section{Instruments, cells and materials}

A potentiostat (273, Princeton Applied Research) interfaced to a computer via GPIB interface (IEEE), along with M270 software (Princeton Applied research), was used for data acquisition and experiment control. This potentiostat was used for obtaining the cyclic voltammetric data and performing the potentiodynamic polarization measurements. A conventional H-shape electrochemical cell equipped with a multiple inlet/outlet system for admission of supporting electrolyte, purging and blanketing the solution with oxygen-free nitrogen, was used. The reference electrode was an $\mathrm{Ag} / \mathrm{AgCl} /\left[\mathrm{Cl}^{-}\right]=1.0 \mathrm{M}$, and all the reported potentials were measured and referenced to this electrode. The working electrode was a $1.0 \mathrm{~mm}$ diameter copper wire $(99.99 \%$ minimum purity, Goodfellow, UK). The immersed part of the wire in the cell was curved in order to provide a mark for obtaining a consistent surface area. The auxiliary electrode was made of platinum (Certified Reagent, 99.99\% minimum purity, Aldrich).

Surface morphology and composition were investigated by SEM (Inspect ${ }^{\mathrm{TM}}$ F50, FEI) equipped with an electron microprobe. 
The square wave with a preset amplitude and frequency was generated by a function generator (BK Precision, 4003). The desired lower and higher limits of the potential regime were obtained by adjusting the applied electrode potential from the potentiostat or the offset of the function generator.

All reagents used were highly pure analytical reagent (A.R.) grade chemicals, and used as received from the suppliers, without further purification. Sulfuric acid (Aldrich, USA) and polyvinylpyrrolidone were supplied by Fluka (USA). The purging nitrogen was G5 Grade, 99.999\% minimum purity, supplied by the International Gas Company, and coupled with Oxisorb ${ }^{\circledR}$ cartridge (Supelco, USA) to remove any residual traces of oxygen. All solutions were made from the above-mentioned reagents dissolved in purified water (Merck Millipore).

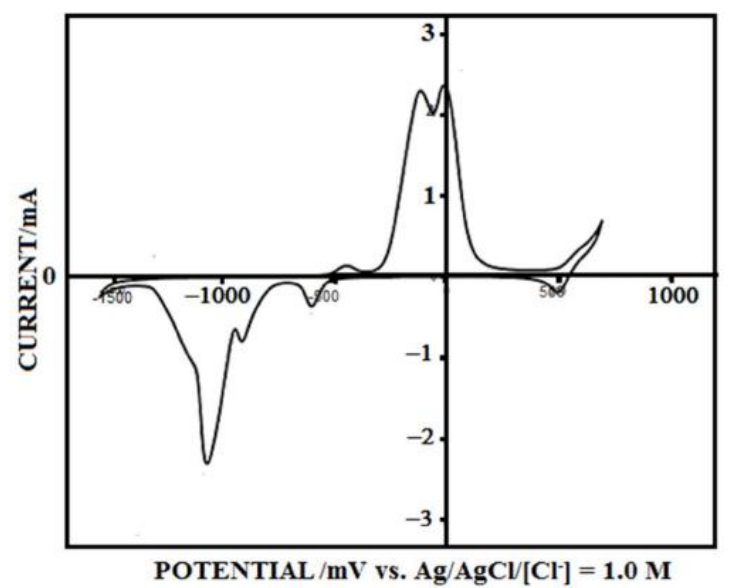

Figure 1. Cyclic voltammogram of a copper electrode in $1.0 \mathrm{M} \mathrm{KOH}$ at a scan rate of $30 \mathrm{mV} / \mathrm{s}$.

\section{Procedures}

Preparation of copper specimens

For cyclic voltammetry and polarization experiments, copper wires were chemically itched by immersion in $20 \% \mathrm{HNO}_{3}$ for $5 \mathrm{~s}$, extensively rinsed with Millipore water, and mounted in the working electrode compartment in an electrochemical cell containing 1.0 M KOH. The electrode was cathodized at $1.2 \mathrm{~V}$ to remove any traces of oxygen from the surface. The surface was conditioned by cycling the electrode potential between -1.5 and $0.745 \mathrm{~V}$. Reproducing a cyclic voltammogram similar to the well-known voltammogram of copper in $1.0 \mathrm{M} \mathrm{KOH}$ was taken as a criterion for copper electrode cleanliness [17]. A representative voltammogram of clean copper electrode under our experimental conditions is displayed in Fig. 1. The voltammogram shows almost all the voltammetric features reported for copper voltammogram under similar experimental conditions. The details of these features are reported elsewhere [17]. Further experimentation was performed on validated-clean copper surfaces.

\section{Weight loss experiments}

Weight-loss methodology for evaluation of the corrosion rate was conducted on 2 $\mathrm{cm}$ long, $1 \mathrm{~mm}$ diameter copper wire specimens. The specimens were pre- 
weighed to the nearest $0.1 \mathrm{mg}$ and placed in an Erlenmeyer flask containing 0.1 $\mathrm{M} \mathrm{NaCl}$ acidified with sulfuric acid to make a final $\mathrm{pH}$ of 2 . The solutions were saturated with oxygen by continuous bubbling of oxygen into the solutions. The sulfuric acid-chloride solution was chosen as a corrosive medium, because, at one hand, sulfuric acid is widely used in copper plating industry, and, at the other, because the N-heterocyclic compounds are known as efficient inhibitors in sulfuric acid containing chloride ions [15].

Copper specimens were subjected to a square wave potential regime, with a set of preset experimental conditions (i.e., frequency, amplitude, upper and lower values of the square wave, and concentration of PVP).

The pre-weighed copper specimens were immersed in the above-mentioned corrosive medium for $72 \mathrm{~h}$. At the end of the test period, the specimens were rinsed, dried, and re-weighed on the analytical balance.

Corrosion rate determination was conducted on two groups of samples. The first was the control group, where copper specimens were exposed to the inhibitor for $72 \mathrm{~h}$. The second group included specimens which were subjected to square wave potential regimes in solutions containing PVP.

\section{Electrochemical corrosion measurements}

Electrochemical measurements involved three types of experiments. The first type involved reproducing the voltammogram of copper as an "electrochemical signature" for a clean non-oxidized polycrystalline copper surface. The second type of experiments involved investigation of adsorption of PVP at the copper electrode. The third type was concerned with polarization curves which were needed for calculation of the corrosion rate for the specimens subjected to the square wave potential regime and the control specimens. Electrochemical measurements were conducted in $1.0 \mathrm{M} \mathrm{KOH}$ solutions, because the voltammetric features are well-established in alkaline media rather than in acidic media [17].

\section{Results and discussion}

\section{Adsorption of PVP on polycrystalline copper electrodes}

Fig. 2 shows the cyclic voltammograms of copper polycrystalline electrode in a 1.0 $\mathrm{M} \mathrm{KOH}$ solution in addition to a variable concentration of PVP. The voltammograms display all the well-known reported voltammetric features of polycrystalline copper recorded in a $1.0 \mathrm{M} \mathrm{KOH}$ solution. This indicates that PVP does not alter the surface energy states of copper, but merely adsorbs and blocks a part of the electrode surface.

The voltammograms (Fig. 2) show a monotonic decrease in the charge underneath all the peaks on the voltammograms with an increased concentration of PVP. This can be attributed to the decrease in the real surface area of the copper electrode. This, in fact, is a consequence of the adsorption of PVP molecules onto copper surface. This also explains in part the mechanism through which PVP inhibits corrosion. Recording the voltammograms in a PVP-free 1.0 $\mathrm{KOH}$ solution, after exposure to the solutions containing PVP showed 
voltammograms that are identical to those recorded in the presence of PVP in the solutions. This observation indicates that PVP is stable on the copper surface and survives rinsing with purified water or a supporting electrolyte solution. This finding is a common belief that corrosion inhibitors inhibit corrosion by adsorption onto metal surfaces [14].

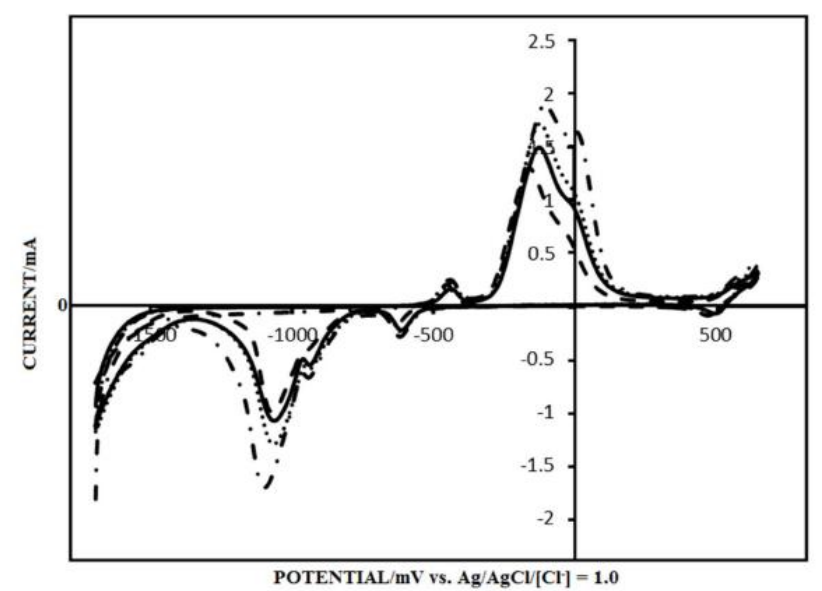

Figure 2. Cyclic voltammograms of copper electrode in $1 \mathrm{M} \mathrm{KOH}$ after exposure to a

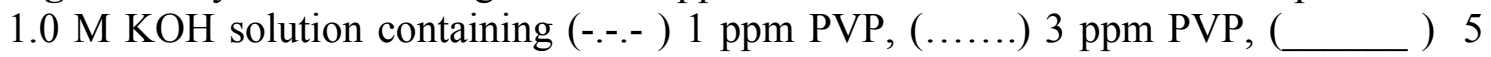
ppm PVP and (- - - ) $10 \mathrm{pm} \mathrm{PVP.} \mathrm{dE} / \mathrm{dt}=30 \mathrm{mV} / \mathrm{s}$.

\section{Effect of applied square wave frequency on adsorption of PVP on copper surfaces}

Fig. 3 shows the cyclic voltammograms of a copper electrode, after exposure to PVP, with application of a square wave potential regime at different frequencies, under identical experimental conditions. The voltammograms show a minimum charge underneath the voltammetric peaks of the copper electrode, upon application of a square wave with $500 \mathrm{~Hz}$. Minimum charge underneath the voltammetric peaks is associated with maximum PVP coverage at the electrode surface.

Thus, the $500 \mathrm{~Hz}$ frequency is the optimal frequency for application of square wave potential regimes to copper specimens for corrosion inhibition. The $500 \mathrm{~Hz}$ frequency presents an intermediate frequency between the two other investigated frequencies, $100 \mathrm{~Hz}$ and $1000 \mathrm{~Hz}$.

It is well known that, at high frequencies, the problems of charging current and ohmic potential drop prevent the electrode from acquiring the desired potential. At a lower frequency, $100 \mathrm{~Hz}$, the number of pulses per unit time is lower than those at $500 \mathrm{~Hz}$, which dictates a higher number of molecular rearrangements at the electrode surface. This decrease in the available surface area is expected to enhance corrosion inhibition. 


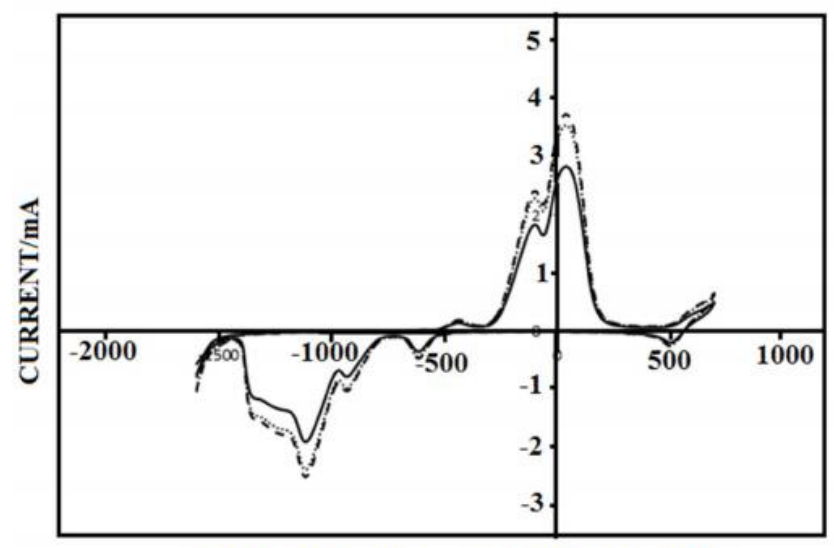

POTENTIAL $/ \mathrm{mV}$ vs. Ag/AgCl$\left[\mathrm{Cl}^{-}\right]=1.0 \mathrm{M}$

Figure 3. Cyclic voltammograms of copper electrode recorded at a scan rate of 30 $\mathrm{mV} / \mathrm{s}$, after application of a square wave potential regime for $60 \mathrm{~s}$ in a $5 \mathrm{ppm}$ PVP solution. The square wave parameters were: $\mathrm{E}_{1}=-1.5 \mathrm{~V}$ and $\mathrm{E}_{\mathbf{h}}=0.7 \mathrm{~V}$ with an amplitude of $2.2 \mathrm{~V}$ centered at $=-0.4 \mathrm{~V}$, with a frequency of $(----) 100 \mathrm{~Hz}$, $500 \mathrm{~Hz}$ and (......) $1000 \mathrm{~Hz}$.

\section{The effect of square wave frequency and exposure time on corrosion rate}

The effect of the square wave on corrosion rate was further explored by recording the polarization curves for plain copper, copper in presence of PVP, and copper in presence of PVP with application of the square wave (Fig. 4 shows a representative example). The corrosion rates were calculated for the three aforementioned surfaces, and the results are given in Table 1. Linear polarization experiments were also conducted on plain copper, copper in presence of PVP and copper in presence of PVP with application of the square wave. From these experiments the polarization resistance was calculated for the three surfaces, and the results are also given in Table 1. The results unequivocally show that there is an enhancement in corrosion inhibition upon application of the square wave to a copper electrode, in the presence of PVP.

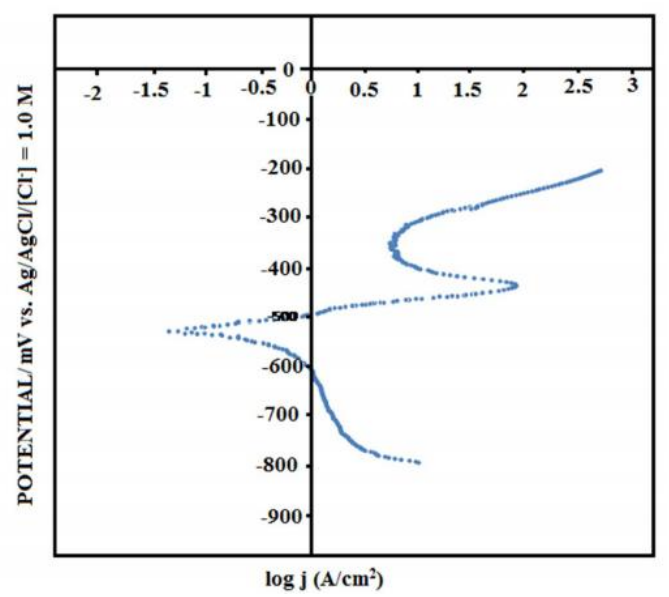

Figure 4. Potentiodynamic polarization curve for copper electrode exposed to a $5 \mathrm{ppm}$ PVP solution in a $1.0 \mathrm{M} \mathrm{KOH}$ solution. 
Table 1. Corrosion potential $\left(\mathrm{E}_{\text {corr }}\right)$, corrosion current $\left(\mathrm{i}_{\text {corr }}\right)$, corrosion rate and corrosion resistance for plain copper, copper in the presence of PVP and copper in the presence of PVP, with application of square wave potential regimes. Square wave parameters: frequency $=500 \mathrm{~Hz}$, time of application of the square wave $=6 \mathrm{~min}$, PVP concentration $=5 \mathrm{ppm}$.

\begin{tabular}{|c|c|c|c|c|}
\hline Specimen & $\mathbf{E}_{\text {corr (V) }}$ & $\mathbf{i}_{\text {corr (A) }}$ & $\begin{array}{c}\text { Corrosion rate } \\
(\mathbf{m p y})\end{array}$ & $\begin{array}{c}\text { Polarization resistance, } \\
\mathbf{\Omega ~ c m}^{-2}\end{array}$ \\
\hline Plain copper & -0.547 & 0.852 & 99.9 & 0.0412 \\
\hline PVP without SW & -0.519 & 0.802 & 94.1 & 0.0741 \\
\hline PVP With 500Hz SW & -0.525 & 0.697 & 81.8 & 0.0793 \\
\hline
\end{tabular}

Moreover, the effect of square wave frequency and exposure time with application of the square wave was evaluated by weight-loss experiments, where the corrosion rates for copper specimens were calculated according to equation 1 :

$$
\text { Corrosion rate }(\text { mpy })=\left(3.45 \times 10^{6} . \mathrm{w}\right) /(\text { A.p.t) }
$$

where mpy is the corrosion rate in mils per year, $\mathrm{w}$ is the weight loss, $\mathrm{A}$ is the geometrical surface area of the electrode, $\rho$ is the density and $t$ is the time of exposure to the inhibitor. The results of these experiments are shown in Fig. 5.

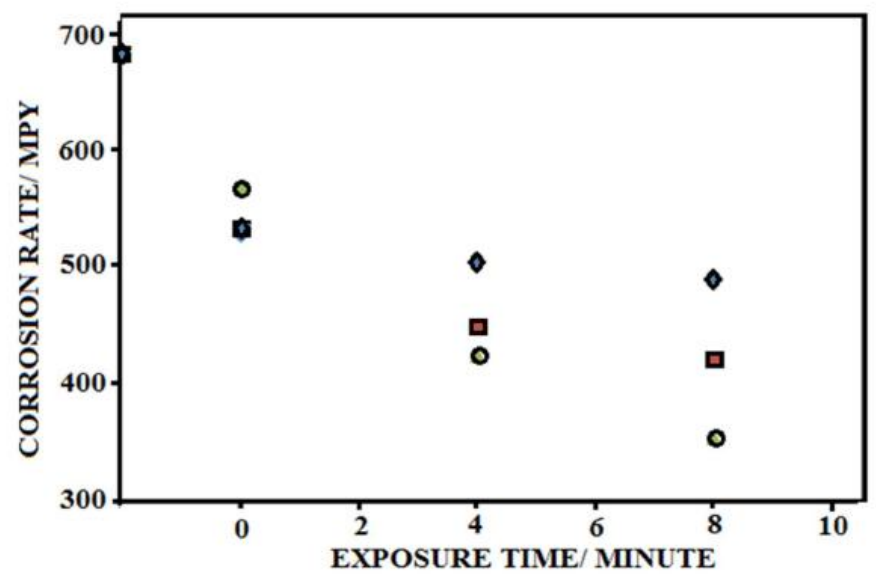

Figure 5. Corrosion rates, as calculated from weight loss experiments for copper substrates, to which a square wave potential regime was applied at $(\boldsymbol{\nabla}) 100 \mathrm{~Hz},(\boldsymbol{\square})$ and (๑) $1000 \mathrm{~Hz}$. Experimental conditions: PVP concentration: 5 ppm, corroding medium: $0.10 \mathrm{M} \mathrm{NaCl}$ adjusted to $\mathrm{pH} 2$. Time for weight loss method $=72$ hours. Square wave parameters: $\mathrm{E}_{\mathrm{l}}=-1.5 \mathrm{~V}, \mathrm{E}_{\mathrm{h}}=-0.7 \mathrm{~V}$, and amplitude $=0.8 \mathrm{~V}$.

Fig. 5 shows a trend of enhancement of corrosion inhibition with an increased time of application of the square wave in presence of PVP. The influence of the increased exposure time on the corrosion rate can be explained as a result of the increased induced adsorption on the copper surface, caused by the application of the square wave (Fig. 2). Explanation of the increased inhibition at $500 \mathrm{~Hz}$ and $1000 \mathrm{~Hz}$ may rely on the increase of the number of adsorption desorption episodes from the surface per unit time with an increased frequency. The number of adsorption/desorption episodes at $1000 \mathrm{~Hz}$, however, is offset by potential drop and charging current problems. This explains the closeness in the 
enhancement of corrosion inhibition upon application of $500 \mathrm{~Hz}$ and $1000 \mathrm{~Hz}$ square wave potential regimes.

\section{Scanning electron microscopy results}

Fig. 6 shows the SEM micrographs for a plain copper electrode, a copper substrate exposed to PVP under open circuit conditions and a copper substrate which was exposed to a PVP solution with the application of a square wave potential regime. The SEM micrographs indicate transformation of PVP on the surface of copper, from scattered dendritic form to a uniform layer covering the surface of copper.

This indicates that the phenomenological influence of the square wave emanates from the increased adsorption coverage of PVP on the copper surface. This might be due to a better alignment of the heteroatom dipoles at the copper surface, as a result of alternating episodes of forward and reverse steps of the square wave.

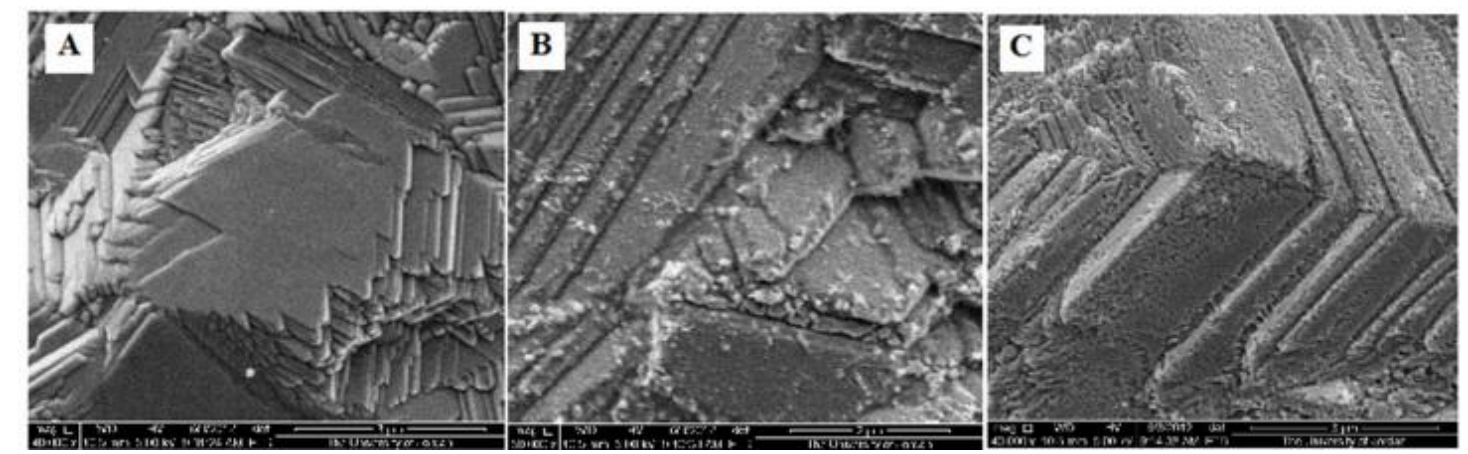

Figure 6. SEM micrographs for (plain copper) copper exposed to a PVP solution at open circuit, and a copper specimen which was subjected to a square wave potential regime in an electrolyte containing PVP. Square wave parameters: frequency $=1000$ $\mathrm{Hz}, \mathrm{E}_{\mathrm{l}}=-1.5 \mathrm{~V}, \mathrm{E}_{\mathrm{h}}=0.7 \mathrm{~V}$, amplitude $=2.2 \mathrm{~V}$, exposure time $=5 \mathrm{~min}$.

\section{Conclusions}

The present work has shown that application of a square wave potential regime to copper substrate decreases the rate of corrosion of copper and promotes inhibition efficiency of PVP. Adsorption of PVP was found to increase monotonically with the application time of the square wave potential regime. Adsorption of PVP at the copper surface was found to survive washing with water, or the supporting electrolyte solution. Rate of corrosion also was found to decrease upon application of square wave potential regimes in a PVP solution. Enhanced adsorption of PVP on the copper surface, and promotion of corrosion resistance by PVP, with the application of square wave potential regimes, may be explained on basis of enhanced PVP adsorption coverage induced by application of the square wave potential regime.

\section{References}

1. Bard A, Faulkner L. Electrochemical methods: fundamentals and applications. 2nd ed. New York; 2001.

2. Hourani M, Wieckowski A. Langmuir. 1990;6:379. 
3. Arvia A, Canullo JC, Custidiano E, et al. Electrochim Acta. 1986;31:1359.

4. Canullo J, Uchida Y, Lehmpfuhl G, et al. Surf Sci. 1987;188:350.

5. Hourani M, Waleed B. J Appl Electrochem. 2000;30:859.

6. Hourani M. J Electroanal Chem. 1994;368:139.

7. Hourani M, Hamdan T. Surf Eng Appl Electrochem. 2013;49:247.

8. Hourani M, Mahmood W. Afinidad. 2013;71:299.

9. Fontana M, Green N. Corrosion engineering. 1st ed. New York: Mc Graw Hill; 1967.

10. Charing T, Lansing F. Corrosion Sci. 1982;42:145.

11. Umoren SA, Eduok UM, Oguzie EE. Port Electrochim Acta. 2008;26:533.

12. Schweinsberg DP, Hope GA, Trueman A. Corrosion Sci. 1996;38:587.

13. Tian H, Li W, Cao K, et al. Corrosion Sci. 2013;73:281.

14. Finšgar M, Milošev I. Corrosion Sci. 2010;52:2737.

15. Thanapackiam P, Rameshkumar S, Subramanian SS, et al. Mater Chem Phys. 2016;174:129.

16. Antonijevic MM, Petrovic MB. Int J Electrochem Sci. 2008;3:1.

17. Ambrose J, Barradas R, Shoesmith D. J Electroanal Chem Interf Electrochem. 1973;47:47. 